\title{
Why Are Antibiotics Prescribed for Patients With Acute Bronchitis? A Postintervention Analysis
}

\author{
William J. Hueston, MD, Julia E. Hopper, Elizabeth N. Dacus, and \\ Arch G. Mainous III, PbD
}

Background: Despite the findings in controlled trials that antibiotics provide limited benefit in the treatment of acute bronchitis, physicians frequently prescribe antibiotics for acute bronchitis. The aim of this study was to determine whether certain patient or provider characteristics could predict antibiotic use for acute bronchitis in a system where antibiotic use had already been substantially reduced through quality-improvement efforts.

Metbods: A retrospective chart review was performed in an academic family medicine training center that had previously instituted a quality-improvement project to reduce antibiotic prescribing for acute bronchitis. Patients who had acute bronchitis diagnosed during an 18-month period and who had no other secondary diagnosis for respiratory distress or a condition that would justify antibiotics were selected from a computerized-record database and included in the study $(n=135)$. Charts were reviewed to document patient symptoms, physical findings, provider and patient characteristics, and treatment.

Results: Thirty-five (26\%) patients received antibiotics for their acute bronchitis. Adults were more likely to receive antibiotics than children $(34 \%$ vs $3 \%, P<.001)$. Analysis of 20 different symptoms and physical findings showed that symptoms and signs were poor predictors of antibiotic use. Likewise, no significant differences were found based on prescribing habits of individual providers or provider level of training.

Conclusion: In a setting where antibiotic use for acute bronchitis had been decreased through an ongoing quality-improvement effort, it did not appear that providers selectively used antibiotics for patients with certain symptoms or signs. Other factors, such as nonclinical cues, might drive antibiotic prescribing even after clinical variation is suppressed. (J Am Board Fam Pract 2000;13:398-402.)

Injudicious antibiotic use for respiratory tract infections has been recognized as a major health problem and associated with the development of multiple-drug-resistant Streptococcus pneumoniae. ${ }^{1-3}$ One common respiratory condition for which antibiotics are frequently prescribed is acute bronchitis. ${ }^{4}$ Antibiotic use for acute bronchitis is common despite several studies that suggest antibiotics are of little to no benefit in the treatment of acute bronchitis. ${ }^{5-7}$ In fact, a recent study examining antibiotic use and acute bronchitis concluded that patients have acute bronchitis diagnosed simply as an excuse to use an antibiotic. ${ }^{8}$ If antibiotics are to be used more judiciously, physicians must be convinced to reduce their prescribing of antibiotics for

Submitted, revised, 29 March 2000.

From the Department of Family Medicine $(\mathrm{WJH}, \mathrm{JEH}$, END, AGM), Medical University of South Carolina, Charleston. Address reprint requests to William J. Hueston, MD, Department of Family Medicine, MUSC, 295 Calhoun St., PO Box 250192, Charleston, SC 29425. Ms. Dacus and Ms. Hopper participated in the Family Medicine Summer Student Externship Program in the Department of Family Medicine. conditions such as acute bronchitis where antibiotics might not be beneficial.

The frequent use of antibiotics and the perceived underutilization of bronchodilators for acute bronchitis were noted by faculty and residents in the Family Medicine Center at the Medical University of South Carolina in the mid-1990s. At that time, antibiotic and bronchodilator use in the Family Medicine Center was similar to national data, with approximately $60 \%$ of patients with acute bronchitis being prescribed antibiotics and fewer than $10 \%$ receiving a bronchodilator. As described in detail elsewhere, during a 14-month period from January 1996 to March 1997, a quality-improvement initiative on acute bronchitis reduced antibiotic use to less than $30 \%$, whereas bronchodilators were prescribed for more than $60 \%$ of patients diagnosed with acute bronchitis. ${ }^{9}$ The project consisted of educational sessions with physicians regarding evidence suggesting improvement of symptoms in patients using bronchodilators and minimal benefits from antibiotics in patients with acute bronchitis. A monthly summary of antibiotic and bronchodilator use was maintained and distrib- 
uted to the providers so they could chart the group's progress at reducing antibiotic use. After the 14-month period, no additional educational efforts were expended; antibiotic and bronchodilator prescribing rates have remained stable since the end of the formal intervention.

Although the quality-improvement project was viewed as a successful intervention, about one quarter of patients were still receiving antibiotics when they were not indicated. The purpose of this study was to determine whether continued antibiotic prescribing for acute bronchitis was associated with certain clinical features of patients (sicker persons), was limited to certain providers (high prescribers), or was a random event. We hoped that describing clinical predictors of antibiotic use would allow a better understanding of physician decision making when physicians encountered patients with acute bronchitis. Such findings might also allow for the development of more successful quality-improvement interventions that could result in even greater reductions in antibiotic use than what had already been accomplished and could be used elsewhere in similar efforts.

\section{Methods}

Patients in this study were selected from the population of patients who receive care at the Department of Family Medicine at the Medical University of South Carolina. At the time of the study, the department provided clinical services at two clinical sites that generated approximately 38,000 ambulatory patient encounters each year. Care for patients seen at the Department of Family Medicine was provided by family practice residents, physician faculty, nurse practitioners, and physician assistants. The protocol for the study was approved by the Institutional Review Board at the Medical University of South Carolina.

Records were searched for all patients with a problem code 466.0 (acute bronchitis) between 1 July 1997, and 31 December 1998, and the resulting charts made up the sample for this study $(\mathrm{n}=$ 165). Charts of patients who had visits that contained a secondary diagnosis of otitis media, sinusitis, asthma, chronic obstructive pulmonary disease, congestive heart failure, or pneumonia were excluded $(n=30)$, leaving a total of 135 charts for review.

Two medical students conducted a detailed chart review of these records. Information of demographics, symptoms, and physical findings were recorded as well as the provider's level of training
Table 1. Characteristics of Patients with Acute Bronchitis Who Received and Did Not Receive Antibiotic Treatment.

\begin{tabular}{|c|c|c|c|}
\hline Characteristics & $\begin{array}{c}\text { Antibiotic } \\
\text { No. (\%) }\end{array}$ & $\begin{array}{l}\text { No Antibiotic } \\
\text { No. (\%) }\end{array}$ & $P$ Value \\
\hline Age & & & $<.001$ \\
\hline$\geq 18$ years & $34(34)$ & $66(66)$ & \\
\hline$<18$ years & $1(3)$ & $34(97)$ & \\
\hline Sex & & & .16 \\
\hline Female & $24(30)$ & $55(70)$ & \\
\hline Male & $11(20)$ & $45(80)$ & \\
\hline Visit in previous 3 months & & & .34 \\
\hline Yes & $6(19)$ & $25(81)$ & \\
\hline No & $29(27)$ & $75(63)$ & \\
\hline Cigarette smoker & & & .95 \\
\hline Yes & $10(26)$ & $28(74)$ & \\
\hline No & $25(26)$ & $72(74)$ & \\
\hline
\end{tabular}

for each record reviewed. Patient age was dichotomized into adults (18 years and older) and children (younger than 18 years). To assess the interrater reliability of the reviewers, both reviewers abstracted a random sample of $10 \%$ of the charts. Interrater agreement was excellent, ranging from $93 \%$ to $100 \%$ depending on the variable.

Bivariate analyses of individual symptoms and physician examination findings with the use of antibiotics were performed using chi-square statistic or the Fisher exact test when expected cell sizes were less than 5 . Because of the large number of demographic variables $(n=4)$, symptom variables $(n=11)$ and physical finding variables $(n=9)$ involved in this study, we used the Bonferroni adjustment for multiple comparisons and calculated a $P$ value of 0.002 (0.05/24 possible comparisons) as the indicator of statistical significance. ${ }^{10}$

\section{Results}

After the conclusion of the quality-improvement intervention, we found that $35(26 \%)$ of the patients with acute bronchitis still received an antibiotic for treatment. When we compared the characteristics of these patients with others who did not receive antibiotics (Table 1), we found that only age appeared to influence the use of antibiotics. Adults were 11 times more likely to receive antibiotics than children $(34 \%$ vs $2.9 \%, P<.001)$. The sex of the patients, smoking status, or whether they had been evaluated for a viral upper respiratory tract infection or acute bronchitis within the last 3 
Table 2. Patient Symptoms and Antibiotic Treatment for Acute Bronchitis.

\begin{tabular}{lcccc}
\hline Symptom & $\begin{array}{c}\text { Antibiotic } \\
(\mathrm{n}=35) \\
\text { No. }(\%)\end{array}$ & $\begin{array}{c}\text { No Antibiotic } \\
(\mathrm{n}=100) \\
\text { No. }(\%)\end{array}$ & $\begin{array}{c}\text { Odds Ratio } \\
(95 \% \text { CI) }\end{array}$ & $P$ Value \\
\hline Chest pain & $9(26)$ & $7(7)$ & $4.60(1.38,15.59)$ & .006 \\
Fever & $12(34)$ & $21(21)$ & $1.96(0.77,5.01)$ & .12 \\
Nausea & $0(0)$ & $1(1)$ & $0.0(0.0,51.63)$ & .99 \\
Runny nose & $4(11)$ & $17(17)$ & $0.63(0.16,2.24)$ & .43 \\
Shortness of breath & $6(17)$ & $8(8)$ & $2.38(0.66,8.50)$ & .19 \\
Sleep disturbance & $3(9)$ & $6(6)$ & $1.47(0.7,7.26)$ & .70 \\
Sore throat & $6(17)$ & $21(21)$ & $0.78(0.25,2.33)$ & .62 \\
Sweats & $3(9)$ & $1(1)$ & $9.28(0.80,244.43)$ & .05 \\
Wheezing & $1(3)$ & $11(11)$ & $0.24(0.01,2.93)$ & .18 \\
\hline
\end{tabular}

${ }^{\star}$ No antibiotic used as reference value.

months did not appear to be predictors of antibiotics use in patients with acute bronchitis.

When we examined patient symptoms and antibiotic use, we found that only chest pain was associated with the use of antibiotics (Table 2). However, even in the patients receiving antibiotics, chest pain was uncommon. Because smoking might occur more often in adults than children, we also examined whether smoking was a predictor of antibiotic use in adult patients (older than 17 years of age). There was no association between smoking status and antibiotic use, however, even when restricted to adults $(P=.34)$. Similarly, there also were no physical examination findings that predicted antibiotic use (Table 3). The most common physical finding described was wheezing, which was seen in $29 \%$ of patients who had a prescription for antibiotics, but was also noted in $30 \%$ of those who did not receive an antibiotic prescription.

We also found no association between provider training and the use of antibiotics, with similar percentages of patients who saw attending physicians $(26 \%)$, residents $(25 \%)$, and midlevel provid- ers $(29 \%)$ receiving antibiotic prescriptions $(P=$ .93) (Table 4). Nor did we find significant variation among individual providers in prescribing antibiotics $(P=.42)$. When we limited analysis to those providers who had diagnosed acute bronchitis in more than 10 patients, we also could not detect any high prescribers whose actions could have skewed data for the entire group.

\section{Discussion}

The results of this study suggest that after a qualityimprovement intervention which reduced antibiotic use for acute bronchitis by more than $50 \%$, antibiotics are prescribed for acute bronchitis more often in adults than children. Except for the patient's age, we could not find any other symptom or physical examination finding that predicted antibiotic use. Physicians could be reserving antibiotics for patients who seem sicker, but because we could measure only the presence of symptoms and signs, not their severity, we could not determine whether such was the case. Physicians also could be making

Table 3. Physical Examination Findings and Antibiotic Treatment for Acute Bronchitis.

\begin{tabular}{|c|c|c|c|c|}
\hline Signs & $\begin{array}{c}\text { Antibiotic } \\
(\mathrm{n}=35) \\
\text { No. }(\%)\end{array}$ & $\begin{array}{c}\text { No Antibiotic } \\
(\mathrm{n}=100) \\
\text { No. }(\%)\end{array}$ & $\begin{array}{l}\text { Odds Ratio } \\
(95 \% \mathrm{CI})^{*}\end{array}$ & $P$ Value \\
\hline Cervical lymphadenopathy & $0(0)$ & $9(9)$ & $0.0(0.0,1.66)$ & .11 \\
\hline Erythema of tympanic membrane & $1(3)$ & $1(1)$ & $2.91(0.0,112.40)$ & .45 \\
\hline Erythematous throat & $5(14)$ & $26(26)$ & $0.47(0.14,1.48)$ & .16 \\
\hline Nasal erythema & $1(3)$ & $10(10)$ & $0.26(0.01,2.18)$ & .29 \\
\hline Sinus tenderness & $2(6)$ & $1(1)$ & $6.00(0.40,176.18)$ & .16 \\
\hline Wheezes in chest & $10(29)$ & $30(30)$ & $0.93(0.36,2.37)$ & .87 \\
\hline
\end{tabular}

${ }^{*}$ No antibiotic used as reference value. 
Table 4. Antibiotic Prescribing Patterns for Different Types of Providers.

\begin{tabular}{lc}
\hline Provider & $\begin{array}{c}\text { Antibiotics Prescribed/ } \\
\text { Total No. Patients } \\
\text { with Acute Bronchitis } \\
\text { No. (\%) }\end{array}$ \\
\hline Resident physicians & $15 / 60(25)$ \\
Attending physicians & $14 / 53(26)$ \\
Nurse practitioner or physician assistant & $6 / 21(29)$ \\
\hline
\end{tabular}

$P=.93$.

their decisions based on other variables that we did not consider or that could not be obtained from the patient record. The issues of whether physicians prescribe antibiotics for these sicker patients or other clinical cues and whether there is any benefit of antibiotics in those who appear more ill remains to be clarified.

Our data also did not support the possibility that some providers were overprescribing antibiotics while others were more judicious. Among those who saw the greatest number of acute bronchitis patients, there was only a modest amount of variation in antibiotic prescribing. This finding suggests that after the quality-improvement intervention, residual antibiotic prescribing was not limited to a few recalcitrant clinicians who did not support the quality-improvement initiative. The intervention appears to have influenced antibiotic prescribing in the entire population of providers, but was only partially successful in that it did not eliminate antibiotic use for all cases of acute bronchitis. It only blunted use by everyone.

In a recent study that prospectively evaluated physician prescribing behavior in a system where antibiotic use for acute bronchitis had not been discouraged, Gonzales and co-workers ${ }^{8}$ found that clinicians appeared to prescribe antibiotics when any of a wide range of clinical features were present. Some of these features, such as purulent nasal discharge, were not even related to bronchitis. The observation that antibiotic use was not necessarily related to symptoms of bronchitis led the investigators to conclude that the diagnosis was used as an excuse to prescribe antibiotics. Our study findings suggest that when it is no longer acceptable to diagnose acute bronchitis as an excuse for using antibiotics, antibiotic prescribing becomes more haphazard and is not dependent on clinical cues. It is unclear whether physicians continue to prescribe antibiotics for the same patients but simply reclassify their condition as another diagnosis, such as acute sinusitis, where antibiotic use will not be questioned.

One drawback of the quality-improvement intervention is that it focused solely on physician perceptions and behaviors and did not address patient desires and expectations. It is possible that once physician attitudes changed, the mechanism driving continued antibiotic use was not the physician's perception but the patient's expectation that the patient needed antibiotics. As previously indicated by a study of antibiotic prescribing in children, ${ }^{11}$ physicians might perceive that certain patients want antibiotics and might prescribe antibiotics to meet this expectation. Others ${ }^{12}$ have shown that when physicians believe a patient wants an antibiotic for cold symptoms, those physicians are more likely to prescribe antibiotic therapy. This same study, however, showed that physicians are poor judges of patient expectations and tend to overestimate patients' desires for antibiotic treatment. Our data would suggest that even after antibiotic prescribing is reduced by a quality-improvement intervention, it would appear that nonclinical issues, such as physicians' perceptions of patient expectations, remain a strong motivation for prescribing antibiotics.

The implications of these findings on additional quality-assurance interventions are not encouraging. These data provide no clear means to intervene on current antibiotic use other than the educational intervention that has already been undertaken. If antibiotic use is a random event and probably connected to nonclinical factors, the intervention most likely to be successful should probably be aimed at patient expectations rather than physician behaviors. Previous research has shown that patients misinterpret the signs and symptoms of colds and believe that antibiotics are justified for conditions that simulate acute bronchitis. ${ }^{13}$ Better education of patients regarding the appropriate use of antibiotics might be more useful than targeting physician knowledge or behaviors.

These conclusions should be interpreted in light of the limitations of the study. First, the study was conducted with a small group of physicians in an academic setting. This group had already undergone a quality-improvement initiative to reduce antibiotic prescribing for acute bronchitis. Physicians had been educated about unjustified antibiotic use for uncomplicated acute bronchitis, and antibiotic prescribing rates had dropped by half during a 15 -month period. Thus, the residual antibiotic use 
found in this study might not reflect antibiotic prescribing patterns by physicians whose antibiotic use had not been scrutinized.

Second, we relied on the medical record to determine patients' symptoms and physical findings. Medical records probably do not capture all the clinical aspects of a patient's condition but rather provide a concise summary filtered through the mind of the clinician. Physicians might have been attending to certain aspects of the patient's condition or physical findings but not reliably documenting these cues. Most physicians, however, record pertinent clinical observations that affect their decision making. Although possible, it would be unlikely that physicians would systematically fail to record the key features they used for their medical decision making.

Additionally, the clinical features of acute bronchitis are not well delineated, and some of the patients in this study could have had respiratory tract conditions other than bronchitis. Rather than use a standardized definition of acute bronchitis to assure that all patients had bronchitis, we relied on the physician's interpretation of the patient's symptoms and signs to make the diagnosis. Physicians could have misdiagnosed acute bronchitis in patients with colds or other respiratory tract conditions, but the intent of this study was to examine physician behavior when they thought a patient had acute bronchitis.

Finally, the small sample size in this study limited the power to detect even modest differences between the patients who received antibiotics and those who did not. Even so, the most commonly encountered symptoms reported by patients who received antibiotics occurred in about only one third of the patients. Had a larger sample size shown that a particular cluster of symptoms was significantly more common for patients who received antibiotics, it would still be a poor discriminator because the sensitivity would be so low.

In summary, we found that after clinicians are educated about avoiding prescribing antibiotics for acute bronchitis, antibiotic prescribing continues for a small number of patients. Patients who receive antibiotics do not appear to be any different from those who do not receive antibiotics. As with other respiratory tract conditions, such as colds, for which antibiotics are overprescribed, ${ }^{14}$ adults are more likely to receive antibiotics than children. These results suggest that such nonclinical factors as patient expectations are the driving forces behind antibiotic overuse. Instead of focusing on phy- sician education, future quality-improvement initiatives directed at reducing consumer demand might be more effective at curtailing antibiotic overuse.

\section{References}

1. Deeks SL, Palacio R, Ruvinsky R, et al. Risk factors and course of illness among children with invasive penicillin-resistant Streptococcus pneumoniae. The Streptococcus pneumoniae Working Group. Pediatrics 1999;103:409-13.

2. Chen DC, McGeer A, de Azavedo JC, Lowe DE. Decreased susceptibility of Streptococcus pneumoniae to fluoroquinolones in Canada. N Engl J Med 1999; 341:233-9.

3. Austin DJ, Kristinsson KG, Anderson RM. The relationship between the volume of antimicrobial consumption in human communities and the frequency of resistance. Proc Natl Acad Sci 1999;96:1152-6.

4. Mainous AG 3rd, Zoorob RJ, Hueston WJ. Current management of acute bronchitis in ambulatory care: the use of antibiotics and bronchodilators. Arch Fam Med 1996;5:79-83.

5. Orr PH, Scherer K, Macdonald A, Moffatt MEK. Randomized placebo-controlled trials of antibiotics for acute bronchitis: a critical review of the literature. J Fam Pract 1993;36:507-12.

6. Smucny JJ, Becker LA, Glazier RH, McIsaac W. Are antibiotics effective treatment for acute bronchitis? A meta-analysis. J Fam Pract 1998;47:453-60.

7. Fahey T, Stocks N, Thomas T. Quantitative systematic review of randomised controlled trials comparing antibiotic with placebo for acute cough in adults. BMJ 1998;316:906-10.

8. Gonzales R, Barrett PH Jr, Crane LA, Steiner JF. Factors associated with antibiotic use for acute bronchitis. J Gen Intern Med 1998;13:541-8.

9. Ornstein SM, Jenkins RG, Lee FW, Sack JL, LaKier EI, Roskin SD, Wulfman JS, Wriston GA. The computer-based patient record as a CQI tool in a family medicine center. Jt Comm J Qual Improve 1997;23: $347-61$.

10. Mathews DE, Farewell VT. Using and understanding medical statistics. Farmington: S. Karger AG, 1988.

11. Vinson DC, Lutz LJ. The effect of parental expectation on the treatment of children with a cough: a report from ASPN. J Fam Pract 1993;37:23-27.

12. Hamm RM, Hicks RJ, Bemben DA. Antibiotics and respiratory infections: are patients more satisfied when expectations are met? J Fam Pract 1996;43:56-62.

13. Mainous AG 3rd, Zoorob RJ, Oler MJ, Haynes DM. Patient knowledge of upper respiratory infections: implications for antibiotic expectations and unnecessary utilization. J Fam Pract 1997;45:75-83.

14. Mainous AG, Hueston WJ, Clark JR 3rd. Antibiotics and upper respiratory infections. Do some folks think there is a cure for the common cold? J Fam Pract 1996;42:357-61. 